\title{
Poly(isobutylcyanoacrylate) nanoparticles decorated with chitosan: Effect of conformation of chitosan chains at the surface on complement activation properties
}

\author{
Christelle Zandanel ${ }^{1,2}$; Christine Vauthier ${ }^{1,2, *}$
}

1. Univ Paris-Sud, Physico-chimie, Pharmacotechnie, Biopharmacie, UMR CNRS 8612, Chatenay Malabry, F-92296.

2. CNRS, Chatenay Malabry, F-92296.

Submited : February 15, Accepted : March 3

Published in: J. Coll Sci Biotechnol (Journal of Colloid and Biotechnology) 1: 68-81 (2012). doi:10.1166/jcsb.2012.1004

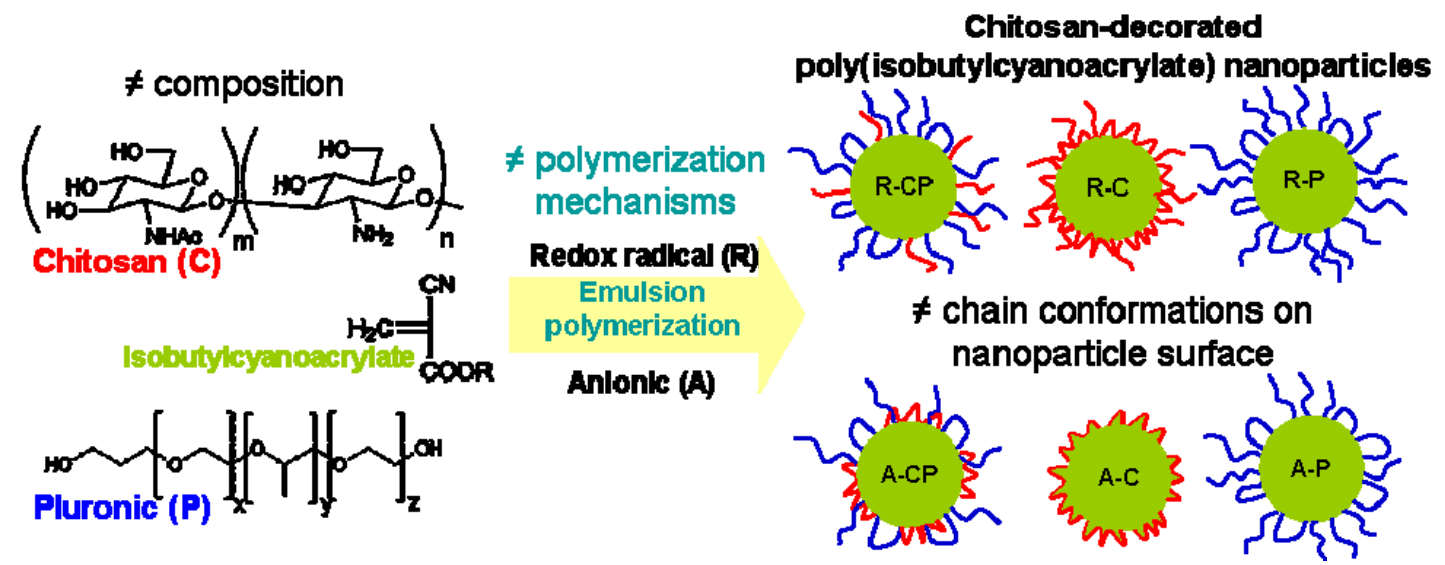

Structures of polymers composing nanopartides suitable for in vivo delivery of siRNA were elucidated. Models illustrating the conformational taken by chitosan chains on the nanoparticle surface were established and correlated well with complement activation profileshown by thenanopartides. Resultswill behelpful to designstealth and safenanopartidesavoidingcomplementactivation.

\begin{abstract}
Poly(alkylcyanoacrylate) nanoparticles decorated with chitosan were found interesting drug carriers to deliver siRNA (small interfering RNA) to tumor by intravenous injection. The nanoparticles are prepared by emulsion polymerization in presence of chitosan and pluronic ${ }^{\circ} \mathrm{F} 68$. The present work was aimed to study the structure of polymers composing the nanoparticles to obtain useful information about the structure of the nanoparticle surface. It was based on the characterization of nanoparticles obtained from polymerization media containing or not pluronic ${ }^{\circ} \mathrm{F} 68$ and cerium. It was shown that the structure of copolymers composing the nanoparticles greatly depended on the predominant mechanism of polymerization governed by the composition of the polymerization medium. Models of the conformation taken by the hydrophilic chains (chitosan and pluronic ${ }^{\circledR} F 68$ ) at the surface of the nanoparticles could be suggested from the structures of copolymers composing the different nanoparticles. A clear correlation of the structure of the nanoparticle surface with the capacity of the nanoparticles to trigger the activation of the complement system was established. These results will be useful to design nanoparticles with a low capacity to activate the complement system which is required to obtain stealth and safe nanoparticles.
\end{abstract}

* Corresponding author: Christine VAUTHIER. Laboratoire de Physico-chimie, Pharmacotechnie, Biopharmacie, UMR CNRS 8612, Faculté de Pharmacie, 5 rue J.B. Clément, 92296 Chatenay Malabry (France) E-mail: christine.vauthier@u-psud.fr, Phone: + 331468356 03; Fax: + 33146835312 


\section{Introduction}

Polymer nanoparticles are developed as drug carriers to improve efficacy of drug delivery by targeting high concentrations to the diseased tissue. ${ }^{1}$ For the success of the method, it is required that the nanoparticles are safe and show a perfectly controlled biodistribution. From previous works, it was identified that characteristics of nanoparticles influencing their in vivo fate after an intravenous administration were their size, surface charge and shape. ${ }^{2,3,4}$ In general, these parameters are included in most studies. However, the few occurrences of unacceptable immune reactions responsible for patient death reported after treatments with stealth nanomedicines ${ }^{5}$ suggested that this description of nanoparticles is insufficient. ${ }^{2,5,6}$ It was established that the immune reaction resulted from a strong activation of the complement system triggered by the nanoparticles. ${ }^{5,7} \mathrm{~A}$ better understanding on the relation between the nanoparticle structure and the capacity to trigger the activation of the complement system is now expected to improve the design of safer nanoparticulate drug carriers. A first step to the establishment of this relation includes a stronger characterization of nanoparticles.

Over the last 15 years, nanoparticles for in vivo drug delivery were developed on the basis of a corecorona structure using amphiphilic copolymers. The core is made of a hydrophobic polymer. The corona, which hides the core, is composed of hydrophilic polymers. A few studies considered the influence of the characteristics and structure of the corona on the interactions of nanoparticles with proteins. ${ }^{4,8,9,10,11}$ It was reported that interactions of nanoparticles with proteins including proteins of the complement system were highly sensitive to the structural characteristics of the nanoparticle corona. Most nanoparticles described in the literature were designed with a corona of poly(ethylene glycol) using block copolymers of known composition and structures., ${ }^{4,12,13}$ Fine structure of nanoparticles can be assumed from the structures of copolymers used for their preparation. Polysaccharides were suggested as alternative components to constitute the corona of the nanoparticles. ${ }^{14,15,16}$ Our group have developed a series of poly(alkylcyanoacrylate) nanoparticles decorated with corona of polysaccharides., ${ }^{9,10,17}$ The nanoparticles are produced by emulsion polymerization of alkylcyanoacrylate implying that polymers form in the same time than nanoparticles. Using this method of preparation of nanoparticles, only a detailed analysis of the composition and structure of polymers make possible description of the nanoparticle structure. Dextran-coated poly(isobutylcyanoacrylate) (PIBCA) nanoparticles obtained by emulsion polymerization were characterized in a previous work. It was highlighted that small changes introduced in the composition of the polymerization medium generated copolymers of different structures. As a consequence, the structure of the corona between the nanoparticles was also different explaining the very different capacities to trigger the activation of the complement system reported by the corresponding nanoparticles. ${ }^{17}$

The aim of the work presented in this paper was to characterize PIBCA nanoparticles decorated with a blend of chitosan and pluronic ${ }^{\circ}$ and to evaluate their capacity to trigger complement activation. These nanoparticles were found suitable to deliver siRNA to tumor after systemic administration to mice. ${ }^{18,19}$ Chitosan was used to promote association of siRNA with the nanoparticles. Pluronic ${ }^{\circ}$ F68 was added to obtain small enough nanoparticles to target a tumor by an enhanced permeation and retention (EPR) effect. ${ }^{19}$ The composition of the polymerization medium used to produce these nanoparticles was rather complex compared to that used for the preparation of the previously characterized dextran-decorated nanoparticles. The conditions of reaction were designed to promote a radical polymerization. However, the high reactivity of the isobutylcyanoacrylate monomer could not exclude the occurrence of a competing anionic polymerization..$^{20}$ Competitions between different polymerization mechanisms can generate various types of polymers. To understand and analyze the role of each component of the polymerization medium on both the radical and anionic polymerization reaction it was decided to perform additional preparations of nanoparticles in polymerization media of much simpler composition. This was expected to reveal the various types of polymers that could be generated in the complex polymerization conditions used to prepare the chitosan-pluronic decorated nanoparticles of interest for in vivo delivery of siRNA. Although this work was intended to provide with a detail characterization of the nanoparticles of interest, the further analysis of their complement activation capacity was expected to explain their remarkable capacity to deliver active siRNA to tumor in vivo. Because nanoparticles prepared from simpler polymerization media will also be characterized in detail, this work was also expected to make 
possible a comparison between several nanoparticle structures and their capacity to trigger activation of the complement system. These results would be useful to identify types of nanoparticles which present a risk to trigger unwanted immune reactions in patients. Complementary, results obtained from nanoparticles showing a low complement activation capacity would help to design stealth and a priori safe nanoparticles.

\section{Materials and methods}

\subsection{Material}

Isobutylcyanoacrylate (IBCA) was purchased from Orapi (Paris, France). Chitosan (medium viscosity), dimethyl sulfoxide- $\mathrm{d}_{6}\left(\mathrm{DMSO}-\mathrm{d}_{6}\right)$, deuterium oxide $\left(\mathrm{D}_{2} \mathrm{O}\right)$ and acetic acid- $\mathrm{d}_{4}$ were purchased from Aldrich. Cerium (IV) ammonium nitrate and nitric acid were purchased from Fluka. Pluronic ${ }^{\circ} \mathrm{F} 68$ was provided as a gift by BASF. Tetrahydrofuran (THF) was purchased from Carlo Erba.

Poly(isobutylcyanoacrylate) was obtained by pooring $250 \mu \mathrm{l}$ of IBCA in Milli- $\mathrm{Q}^{\circ}$ water under gentle stirring. The polymer which was formed rapidly by anionic polymerization of IBCA was recovered by lyophilization.

Chitosan was hydrolyzed in conditions described by Huang and al. ${ }^{21}$ modified by Bertholon et al. ${ }^{20}$ and De Martimprey et al. ${ }^{19}$ with a concentration in sodium nitrite of $11 \mathrm{~g} / \mathrm{L}$. Chitosan used for the synthesis of the nanoparticles had a molecular weight of $21 \pm 4 \mathrm{KDa}$ as evaluated by the measurement of the intrinsic viscosity ${ }^{22}$ and a degree of deacetylation of $80 \%$ as estimated by the ${ }^{1} \mathrm{H}$ NMR. ${ }^{23}$

\subsection{Preparation of nanoparticles}

Two methods of polymerization were used to prepare the different types of nanoparticles. In each method, protocols only differed by the addition of either chitosan or pluronic ${ }^{\circ}$ F68 or both in the polymerization medium used.

Method $\boldsymbol{R}$ (redox radical emulsion polymerization): The required amount of chitosan ( 0 or $0.136 \mathrm{~g}$ ) and pluronic ${ }^{\circ} \mathrm{F} 68(0$ or $3.5 \% \mathrm{w} / \mathrm{v})$ were dissolved in a solution of nitric acid $0.2 \mathrm{~N}(8 \mathrm{~mL})$ at $40-41^{\circ} \mathrm{C}$ under vigorous magnetic stirring and nitrogen atmosphere. After $10 \mathrm{~min}$, a solution of cerium (IV) ammonium nitrate $80 \mathrm{mM}(2 \mathrm{~mL})$ in nitric acid $0.2 \mathrm{M}$ and IBCA $(0.5 \mathrm{~mL})$ were successively added. The bubbling was stopped and the polymerization was continued for $50 \mathrm{~min}$. The latex recovered was cooled down with an ice bath to avoid aggregation.

Method A (anionic emulsion polymerization): The required amount of chitosan (0 or $0.136 \mathrm{~g}$ ) and pluronic ${ }^{\circ} \mathrm{F} 68(0$ or $3.5 \% \mathrm{w} / \mathrm{v})$ were dissolved in a solution of nitric acid $0.2 \mathrm{~N}(8 \mathrm{~mL})$ at $40{ }^{\circ} \mathrm{C}$ under a vigorous magnetic stirring. After $10 \mathrm{~min}, \mathrm{IBCA}(0.5 \mathrm{~mL})$ was added. The bubbling was stopped and the polymerization was continued for $24 \mathrm{~h}$. The latex recovered was cooled down with an ice bath to avoid aggregation.

A systematic nomenclature to name the different nanoparticles was applied. The first letter either $\mathrm{R}$ (i.e., radical) or A (i.e., anionic) indicates the polymerization method used. The addition of chitosan and pluronic ${ }^{\circ} \mathrm{F} 68$ in the polymerization medium was indicated by the letters $\mathrm{C}$ and $\mathrm{P}$ respectively. All nanoparticles considered in the present work are indicated in Table 1.

\subsection{Purification of Nanoparticle dispersions}

After cooling, all suspensions were purified by dialysis using a Spectra/Por membrane with molecular weight cut off $100000 \mathrm{~g} / \mathrm{mol}$, three times during $1 \mathrm{~h}$ and once overnight against $1 \mathrm{~L}$ of water. The suspension was then centrifuged $\left(14 \mathrm{~h}, 4{ }^{\circ} \mathrm{C}, 27440 * \mathrm{~g}\right.$, rotor 70.1 . Ti, Beckman). It was checked by dynamic light scattering that no nanoparticles remained in the supernatant. The concentration of pluronic $\mathrm{F} 68$ was then evaluated by elemental analyses. After removing the supernatant, the pellet containing the nanoparticles was washed with Milli- ${ }^{\circ}$ water by five vortex stirring of 1 min each. $A$ second centrifugation was performed $\left(14 \mathrm{~h}, 4{ }^{\circ} \mathrm{C}, 27440^{*} \mathrm{~g}\right.$, rotor 70.1 . Ti, Beckman) to recover the washed nanoparticles in the pellet. The washed nanoparticles were then lyophilized for $48 \mathrm{~h}$ (Alpha 2-4 LD plus, Bioblock Scientific) after freezing at $-80^{\circ} \mathrm{C}$ for $12 \mathrm{~h}$. The lyophilized nanoparticles were further used to analyze the polymer composition and structure. 
Table 1. Conditions of polymerization and characterization of the size of nanoparticles as evaluated by dynamic light scattering

\begin{tabular}{|c|c|c|c|}
\hline $\begin{array}{c}\text { Name of } \\
\text { nanoparticles }\end{array}$ & $\begin{array}{l}\text { Macromolecules in the } \\
\text { polymerization } \\
\text { medium }\end{array}$ & Polymer & $\begin{array}{l}\text { Nanoparticles } \\
\text { diameter }(\mathrm{nm})\end{array}$ \\
\hline R-CP & chitosan-pluronic F68 & \multirow{3}{*}{ latex } & $81 \pm 20$ \\
\hline R-C & chitosan & & $213 \pm 15$ \\
\hline R-P & pluronic F68 & & $105 \pm 14$ \\
\hline $\mathbf{R}$ & 1 & precipitate & l \\
\hline A-CP & chitosan-pluronic F68 & \multirow{3}{*}{ latex } & $48^{\mathrm{a}}$ \\
\hline A-C & chitosan & & $204 \pm 27$ \\
\hline A-P & pluronic F68 & & $36^{\mathrm{a}}$ \\
\hline $\mathbf{A}$ & 1 & precipitate & 1 \\
\hline
\end{tabular}

\subsection{Methods}

\subsubsection{Size of nanoparticles and concentration of dispersions}

The mean hydrodynamic diameter, $D_{H}$, was evaluated by dynamic light scattering (Nano 90 , Malvern) on suspensions obtained after synthesis and purification by dialysis. The suspensions $(60 \mu \mathrm{L})$ were diluted in acetic acid $0.1 \mathrm{~N}(1 \mathrm{~mL})$ prior to the measurements and the errors were evaluated by the half width peak distribution.

Concentrations in nanoparticles in the dispersions were evaluated by gravimetric measurements. Define aliquots of corresponding nanoparticle suspensions were lyophilized (Alpha 2-4 LD plus, Bioblock Scientific) and the mass of the dried residue was determined.

\subsubsection{Transmission electronic microscopy}

Transmission Electron Microscopy (TEM) was performed using a Philips EM208 with a large format CCD camera AMT at the CCME Orsay (Centre Commun de Microscopie Electronique) France. Samples were diluted in Milli- ${ }^{\circ}$ water 1:100 and deposited on a preionized Formvar-Carbon-coated Electron Microscope Grid. After $5 \mathrm{~min}$, the sample was stained with neutral $1 \%$ aqueous phosphotungstic acid for $30 \mathrm{~s}$ or $1 \mathrm{~min}$. The excess of liquid was removed and the grid was left to dry before observation.

\subsubsection{Solubilization of the nanoparticles in DMSO, in THF and in a blend of THF and water}

Lyophilized nanoparticles $(20 \mathrm{mg})$ were dissolved in DMSO $(1 \mathrm{~mL})$ at $20{ }^{\circ} \mathrm{C}$ under agitation using a vortex for $1 \mathrm{~min}$.

Using THF as a solvent, lyophilized nanoparticles $(25 \mathrm{mg}$ ) were let to dissolve in $2.5 \mathrm{~mL}$ of THF for 3 days at room temperature. The fraction of polymer soluble in THF was then isolated in the supernatant obtained after centrifugation $\left(10 \mathrm{~min}, 10^{\circ} \mathrm{C}, 10000^{*} \mathrm{~g}\right.$, rotor FA-45-30-11, Eppendof 5417R). The molecular weight of the polymer dissolved in the supernatant was then analyzed by size exclusion chromatography in THF (SEC). The remaining undissolved polymer harvested in the pellet was dried at room temperature for $24 \mathrm{~h}$ and the dried residue was weighted. The weight ratio between the soluble and insoluble parts of polymer composing the nanoparticles was deduced from the amount of polymer introduced in THF and the amount of insoluble fraction recovered after the assay of dissolution in THF.

Finally, THF soluble polymers obtained from the dissolution of $5 \mathrm{mg}$ of lyophilized nanoparticles R-CP and R-P in $300 \mu \mathrm{L}$ THF were tested for their solubility in blends of THF and water. To the limpid solution, aliquots consisting of $20 \mu \mathrm{L}$ of water were added until turbidity appeared. The same protocol was applied to a solution of PIBCA homopolymer dissolved in THF.

\subsubsection{Recovery of chitosan and/or pluronic ${ }^{\circ}$ F68 from nanoparticles:}

Hydrolysis of nanoparticles with sodium hydroxyde in aqueous media: the fraction of chitosan/pluronic ${ }^{\circ}$ F68 included in the polymer was recovered after basic hydrolysis of the PIBCA 
part. A sample of lyophilized nanoparticles $(50 \mathrm{mg})$ was stirred in sodium hydroxide solution $(5 \mathrm{~mL}$, $1 \mathrm{~N}$ ) at room temperature during $5 \mathrm{~h}$. The resulted orange solution was totally limpid and the polymer entirely dissolved. This solution was then dialyzed three times against $500 \mathrm{~mL}$ of Milli-Q ${ }^{\circledR}$ water (Spectra/Por membrane, MWCO $500 \mathrm{~g} / \mathrm{mol}$ ). The purified solution collected after dialysis was then lyophilized. The dried residue was dissolved in Milli- $Q^{\circ}$ water $(10 \%(w / v))$ to give a solution which was then filtered through SPE cartridge (positive resin $\mathrm{NH}_{3}{ }^{+}$, SPEC.SAX $3 \mathrm{~mL}$ columns, Interchim). The filtrate which was expected to contain chitosan and pluronic ${ }^{\circ}$ F68 recovered after hydrolysis of the PIBCA part was analyzed by ${ }^{1} \mathrm{H}$ NMR and elemental analysis. To control that chitosan and pluronic ${ }^{\circ} \mathrm{F} 68$ were not affected by the basic hydrolysis, the same procedure was also applied to a solution of chitosan and to a solution of pluronic ${ }^{\circ} \mathrm{F} 68$.

Hydrolysis of nanoparticles with sodium hydride in THF: the fraction of chitosan/pluronic ${ }^{\circ} \mathrm{F} 68$ included in the polymer was recovered after basic hydrolysis of the PIBCA part in an organic solvent. A sample of lyophilized nanoparticles $(40 \mathrm{mg})$ was stirred in THF $(4 \mathrm{~mL})$ at $0{ }^{\circ} \mathrm{C}$ under argon atmosphere. $\mathrm{NaH}(40 \mathrm{mg})$ was slowly added. The inert atmosphere was maintained for $30 \mathrm{~min}$. The reaction mixture was then stirred for $1 \mathrm{~h} 30$ at room temperature. $3 \mathrm{~mL}$ of Milli- $\mathrm{Q}^{\circ}$ water was added at $0^{\circ} \mathrm{C}$ to hydrolyze the remaining $\mathrm{NaH}$ before dialysis. The resulted yellow solution was then dialyzed four times against $500 \mathrm{~mL}$ of Milli- $Q^{\circ}$ water (Spectra/Por membrane, MWCO $500 \mathrm{~g} / \mathrm{mol}$ ). The purified solution recovered after dialysis was then lyophilized. The dried residue was dissolved in Milli- $\mathrm{Q}^{\circ}$ water $(10 \%(\mathrm{w} / \mathrm{v}))$ and the solution was filtered through SPE cartridge (positive resin $\mathrm{NH}_{3}{ }^{+}$, SPEC.SAX $3 \mathrm{~mL}$ columns, Interchim). Filtrate which was expected to contain chitosan and pluronic ${ }^{\circ} \mathrm{F} 68$ recovered after hydrolysis of the PIBCA was analyzed by ${ }^{1} \mathrm{H}$ NMR and elemental analysis.

\subsubsection{Recovery of the PIBCA from nanoparticles:}

The fraction of PIBCA included in the polymer was recovered from lyophilized particles after degradation of the chitosan part by acidic hydrolysis. Lyophilized nanoparticles $(70 \mathrm{mg})$ were stirred in concentrated hydrochloric acid solution $\left(10 \mathrm{~mL}\right.$, min $35 \%$ ) at $60-65^{\circ} \mathrm{C}$ for $4 \mathrm{~h}$. The remaining insoluble part was carefully filtered off with a filter glass $n^{\circ} 3$ and extensively washed with water. The insoluble residue was then recovered by solubilization in THF ( $5 \mathrm{~mL}$ ). A co-precipitation with water (2 $\mathrm{mL}$ ) was performed and THF was removed by evaporation at room temperature for $24 \mathrm{~h}$. The final residue was lyophilized during $48 \mathrm{~h}$. The composition of this fraction was analyzed by ${ }^{1} \mathrm{H}$ NMR in DMSO and the molecular weight evaluated by size exclusion chromatography in THF (SEC).

\subsection{Methods of analysis}

\subsection{1 ${ }^{1} \mathrm{H}$ NMR Spectroscopy}

${ }^{1} \mathrm{H}$ NMR spectra were recorded using a spectrometer $300 \mathrm{MHz}$ (Brucker, Wissenbourg, France). All solutions were prepared at a concentration of $20-25 \mathrm{mg} / \mathrm{mL}$ in DMSO- $d_{6}$ or $D_{2} O$. DMSO- $d_{6}$ was used to dissolve nanoparticles and the PIBCA fraction recovered after the acidic hydrolysis. $\mathrm{D}_{2} \mathrm{O}$ was used to dissolve the insoluble residues recovered after basic hydrolysis and acetic acid- $\mathrm{d}_{4}(10 \mu \mathrm{L})$ was used to dissolve chitosan.

\subsubsection{Elemental analysis}

The composition of the lyophilized nanoparticles was determined by elemental analysis from their content in carbon, nitrogen and hydrogen (Service de microanalyse, Faculté de Pharmacie, Chatenay Malabry, France) using the following equations 1 to 6 giving by the composition of carbon $\mathrm{C}$, nitrogen $\mathrm{N}$, oxygen $\mathrm{O}$ and hydrogen $\mathrm{H}$ :

$$
\begin{aligned}
& C_{\text {total }}=x \times C_{\text {ehttosan }}+y \times C_{E B C A}+z \times C_{\text {pturonte }} \text { e } \\
& N_{\text {total }}=x \times N_{\text {onttasen }}+y \times N_{E B C A}+z \times N_{\text {prumonto }} \\
& Q_{\text {total }}=x \times Q_{\text {ehtrosan }}+y \times Q_{L B C A}+z \times Q_{\text {phronto }}
\end{aligned}
$$




$$
\begin{gathered}
H_{\text {total }}=x \times H_{\text {chttogan }}+y \times H_{L B C A}+z \times H_{\text {pluronte }} \text { s } \\
G_{\text {totai }}+K_{\text {total }}+U_{\text {total }}+H_{\text {total }}=100 \\
x+y+z=1
\end{gathered}
$$

For nanoparticles containing only chitosan, prepared in the absence of pluronic ${ }^{\circ} 68(z=0)$, the content of chitosan, IBCA can be deduced by combining the equations (1) and (6).

For the nanoparticles containing chitosan and pluronic ${ }^{\circ} \mathrm{F} 68$, the content of chitosan, pluronic ${ }^{\circ} \mathrm{F} 68$ and IBCA can be evaluated by combining the equations (1), (3) and (6).

\subsubsection{Dosage of free amine by ninhydrin reagent}

The total content of free amine functions was determined by a colorimetric method with ninhydrin. ${ }^{24,25}$ Reaction between ninhydrin and primary amino group allowed the development of a purple color known as the Ruhemann's purple (detection at $570 \mathrm{~nm}$ ). The secondary amino groups react also with ninhydrin to give a yellow color (detection at $400 \mathrm{~nm}$ ).

For the calibration curve, solutions of glucosamine were prepared in Milli- $\mathrm{Q}^{\circ}$ water at different concentrations $(0 ; 0.9 ; 1.8 ; 2.8 ; 3.7$ and $4.6 \mu \mathrm{mol} / \mathrm{mL})$ from a stock solution $(46.3 \mu \mathrm{mol} / \mathrm{mL})$. Ninhydrin reagent $(10 \mathrm{mg} / \mathrm{mL}$ of acetate buffer $\mathrm{pH} 3.6$ ) was freshly prepared and $1 \mathrm{~mL}$ of the solution was added to the solutions of glucosamine $(1 \mathrm{~mL})$. After stirring, the samples were heated at $90^{\circ} \mathrm{C}$ for $10 \mathrm{~min}$. After the exact heating time, samples were rapidly cooled in an ice bath for $1 \mathrm{~min}$. They were diluted with $15 \mathrm{~mL}$ of Milli-Q ${ }^{\circledR}$ water prior to the measurement of the absorbance at $570 \mathrm{~nm}$ (UV-visible DU 7500 Spectrometer, Beckman). All the absorbance measurements were repeated three times and on several solutions prepared independently. The average calibration curve had the following equation: $\mathrm{Abs}=6376.2^{*} \mathrm{C}$ where $\mathrm{C}$ was the concentration of amine functions given in $\mathrm{mmol} / \mathrm{mL}$.

For application of the dosage to nanoparticles, $1 \mathrm{~mL}$ of the freshly prepared ninhydrine reagent was added to $1 \mathrm{~mL}$ of each suspension. The purple coloration was allowed to develop as described before. After cooling down in an ice bath for $1 \mathrm{~min}$, centrifugation $\left(5 \mathrm{~min}, 4^{\circ} \mathrm{C}, 10000^{*} \mathrm{~g}\right.$, rotor FA-4530-11, eppendorf 5417R) was needed to remove the precipitate of nanoparticles. The absorbance of the supernatant was measured at $570 \mathrm{~nm}$.

The number of free amine functions was determined in $\mathrm{nmol} / \mathrm{mg}$ of polymer by the calibration curve and in $\mathrm{nmol} / \mathrm{mg}$ of chitosan thanks to the percentage of chitosan determined after basic hydrolysis.

\subsubsection{Characterization of polymer molecular weight by steric exclusion chromatography (SEC)}

Molecular weight was determined by steric exclusion chromatography in THF with a double detector (270 dual viscosimeter and light-scattering detector $90^{\circ} \mathrm{C}$, Viscotek, France) and a differential refractometer (Waters 410). THF was used as the mobile phase with a TOSOH BIOSCIENCE TSKgel $5 \mu \mathrm{m}$ bead size Guard precolumn and two TOSOH BIOSCIENCE TSKgel $5 \mu \mathrm{m}$ bead size GMHHR-M columns, operated at $1 \mathrm{~mL} / \mathrm{min}$ with the column temperature set at $30^{\circ} \mathrm{C}$. Samples $(100 \mu \mathrm{L})$ were injected at a concentration in polymer around $10 \mathrm{mg} / \mathrm{mL}$. All samples were centrifuged (10 min, $10{ }^{\circ} \mathrm{C}, 10000 * \mathrm{~g}$, rotor FA-45-30-11, Eppendof 5417R). The supernatant was filtrated over Millipore, PTFE, $0.2 \mu \mathrm{m}$ membranes prior to their injection in the apparatus to remove dust and insoluble residues remained in suspension. The universal calibration of the column was obtained using polystyrene standards. The Omnisec software (version 3.1, Viscotek) was used to process data and recovered the weight average molecular weight $\mathrm{Mw}$.

\subsubsection{Determination of the complement activation profile of the nanoparticles}

Protein C3 is a plasma protein of the complement system circulating in inactive form and involved in the non-specific recognition as opsonization of foreign substances. Activation of the complement system results in the cleavage of the serum protein C3 into smaller fragments including C3a and C3b which can be highlighted by $2 \mathrm{D}$ immunoelectrophoresis using a polyclonal antibody against the 
human C3 protein. ${ }^{26}$ Human serum was obtained after calcifying plasma from healthy donors and stored at $-80{ }^{\circ} \mathrm{C}$ until use. Veronal-buffered saline (VBS), VBS containing $0.15 \mathrm{mM} \mathrm{Ca}^{2+}$ and $0.5 \mathrm{mM}$ $\mathrm{Mg}^{2+}$ ions $\left(\mathrm{VBS}^{2+}\right)$ and VBS containing $40 \mathrm{mM}$ ethylenediaminetetraacetic acid (VBS-EDTA) were prepared as described previously. ${ }^{27}$ Each nanoparticle sample $(100 \mu \mathrm{L})$ at an equal surface area of hydrated nanoparticles corresponding to $400 \mathrm{~cm}^{2}$ were incubated in the presence of human serum $(50 \mu \mathrm{L})$ and $\mathrm{VBS}^{2+}(50 \mu \mathrm{L})$ for $1 \mathrm{~h}$ at $37^{\circ} \mathrm{C}$. Surface area was calculated from the average hydrodynamic diameters according to Vittaz et al. ${ }^{27}$ After incubation, $7 \mu \mathrm{L}$ of each sample was subjected to a first electrophoresis on $1 \%$ agarose gel. The second-dimension electrophoresis was carried out on Gelbonds films in $1 \%$ agarose gel plates (GE Healthcare) containing a polyclonal antibody to human C3 (Complement C3 antiserum rose in goat, Sigma, France), recognizing both C3 and C3b. The films were finally revealed with Coumassie blue to highlight the presence of C3 and C3b which have reacted with the antibody. The results of the complement activation were expressed as a percentage of $\mathrm{C} 3 \mathrm{~b}$ detected on the plate regarding the sum of the peak area of C3 and C3b.

\section{Results}

Alkylcyanoacrylates are extremely reactive monomers. Polymerizations performed in conditions used for the preparation of the nanoparticles can lead different copolymers depending on the composition of the polymerization medium. ${ }^{20}$

In a previous work, the polymerization applied in method R performed with dextran was shown to be mainly initiated thought of the redox radical polymerization discriminating the anionic polymerization mechanism. ${ }^{20}$ However, if the same method of polymerization is carried out with chitosan, the ratio between radical and anionic polymerizations can be modified because of the presence of amine functions in the structure of chitosan. It can further be affected by the addition of pluronic ${ }^{\circ} 68$ in the polymerization medium.

Considering chitosan, it can initiate both anionic and radical polymerization in the polymerization conditions used in method $R$. The radical polymerization can be initiated according to the same mechanism described before with a polymerization medium containing dextran ${ }^{20}$ (Figure 1).

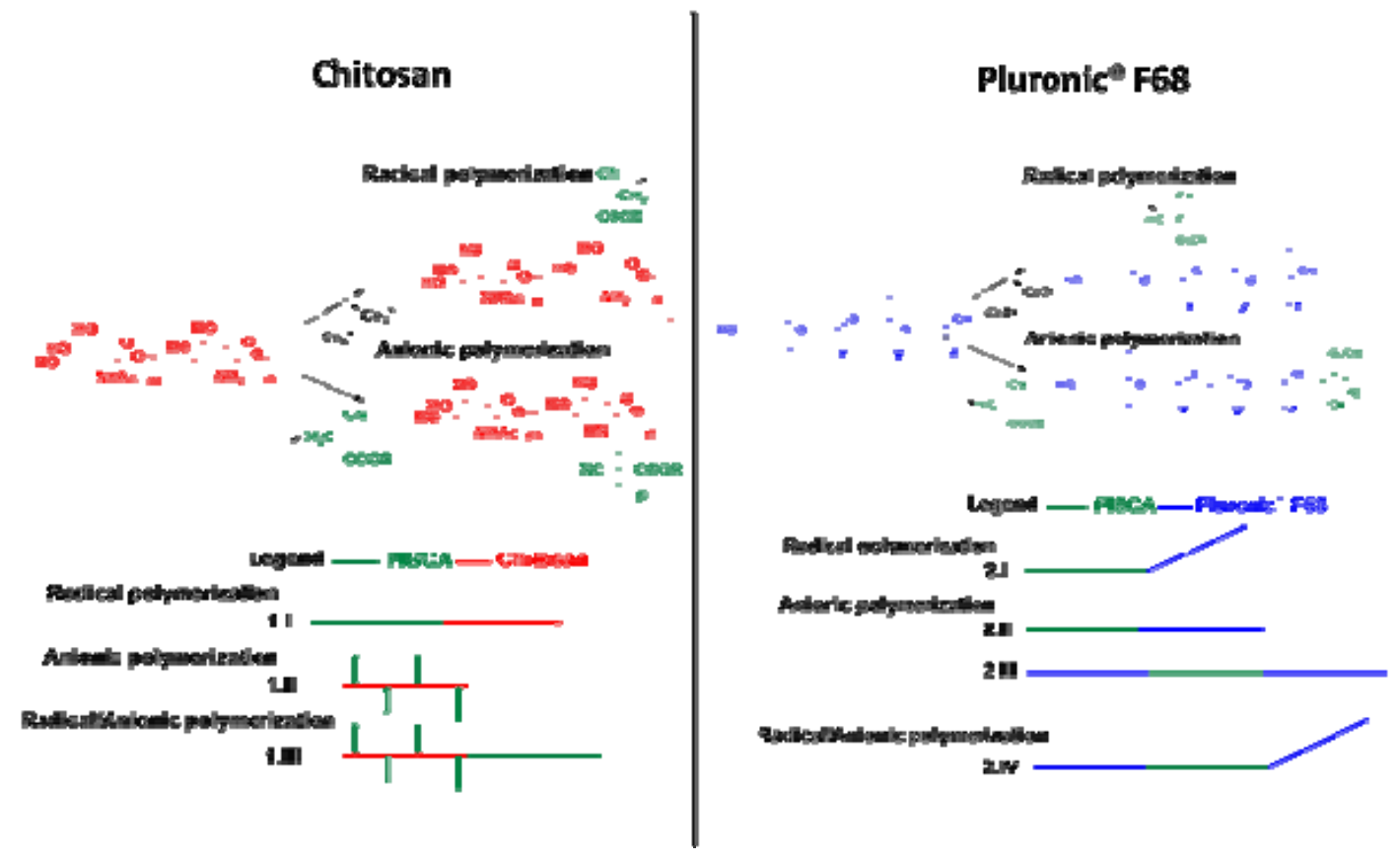

Figure 1: Scheme of the initiation of the polymerization of isobutylcyanoacrylate by chitosan and pluronic 68 according to radical and anionic mechanisms $(A)$ and expected corresponding structures of copolymers (B).

Based on the previous results, it can be assumed that a radical would be created at the end of the chitosan chain thanks to the cleavage of the polysaccharide chain by $\mathrm{Ce}^{4+}$ at the extremely low $\mathrm{pH}$ used in our conditions of polymerization. Indeed, at this $\mathrm{pH}$, the glucosamine residues of the chitosan 
chains are mainly in the open forms. ${ }^{28}$ As shown previously preparing nanoparticles with dextran, at $\mathrm{pH} 1$, the anionic polymerization initiated by hydroxyl groups can be efficiently delayed letting a rapid radical polymerization to take place. ${ }^{16}$ The situation may be different in the presence of chitosan in which reactive amino functions could still initiate polymerization of alkylcyanoacrylates by an anionic pathway (Figure 1). The structures of copolymers which may be expected from the different polymerization mechanisms initiated by chitosan are illustrated in Figure $1 \mathrm{~A}$.

In polymerization medium containing pluronic ${ }^{\circ} \mathrm{F} 68$, the polymerization can also occur according to the two polymerization mechanisms. $\mathrm{Ce}^{4+}$ was reported to generate radicals on the carbon next to the hydroxyl functions end capping the pluronic ${ }^{\circ} \mathrm{F} 68$ chain $^{29,30}$ (Figure 1B). In turn, this radical can initiate a radical emulsion polymerization of IBCA resulting in the formation of a block copolymer with PIBCA. Formation of di-radicals was also reported in the literature, but it seemed that it occurred in a lower extend. ${ }^{30}$ The anionic polymerization of IBCA can be initiated by the free hydroxyl functions (Figure 1). The structures of the different copolymers which may be expected from initiations of those polymerizations are illustrated in Figure 1.

Structures of the polymers composing the nanoparticles prepared in the different conditions used in this work to produce the nanoparticles were characterized according to the scheme presented in Figure 2. Results from this characterization are reported bellow. The capacity of the nanoparticles to trigger the activation of the complement system was also evaluated.

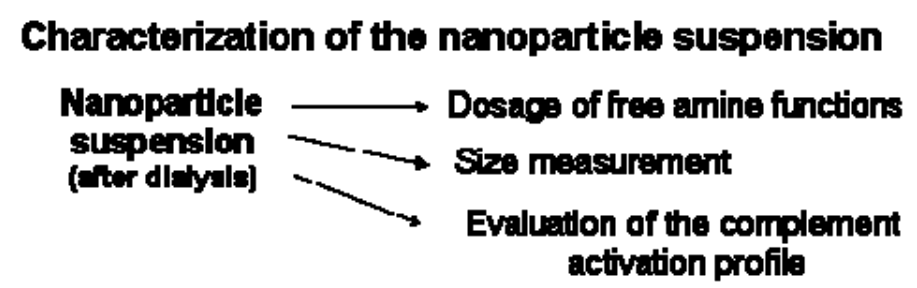

Analysls of the compositlon of the nanoparticles

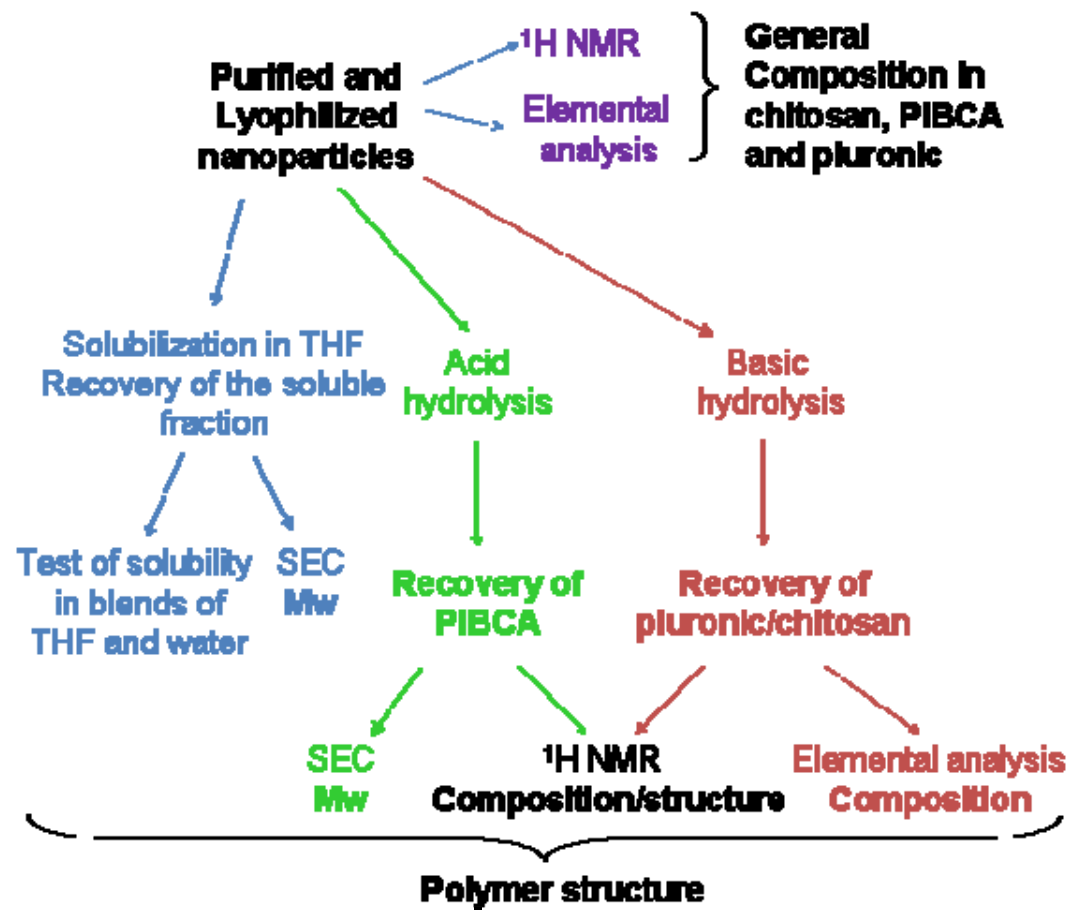

Figure 2. Summary of the different analysis performed on nanoparticles to elucidate composition and structure of copolymers 


\subsection{Size of nanoparticles, transmission electronic microscopy and solubility in different solvents}

When polymerization was performed in the absence of chitosan and pluronic ${ }^{\circ} \mathrm{F} 68$ by methods $A$ and $\mathrm{R}$, a precipitate of polymer was recovered after $24 \mathrm{~h}$ (Table 1 ). In all other conditions, nanoparticles suspensions were formed. The mean hydrodynamic diameters of the nanoparticles are reported in Table 1. Preparations performed with chitosan showed nanoparticles with a diameter around 200 $\mathrm{nm}$. The addition of pluronic ${ }^{\circ} \mathrm{F} 68$ reduced the size of the nanoparticles obtained by both methods in agreement with previous works. Nanoparticles were spherical and presented a core-corona structure shown in transmission electronic microscopy (Figure 3) ${ }^{19}{ }^{\text {Preparations carried out with pluronic }}{ }^{\circ} \mathrm{F} 68$ only led to nanoparticles with much smaller diameters (106 nm for R-P nanoparticles and $40 \mathrm{~nm}$ for A-P nanoparticles). Nanoparticle dispersions A-CP and A-P contained both nanoparticles and aggregates indicated that they were unstable.

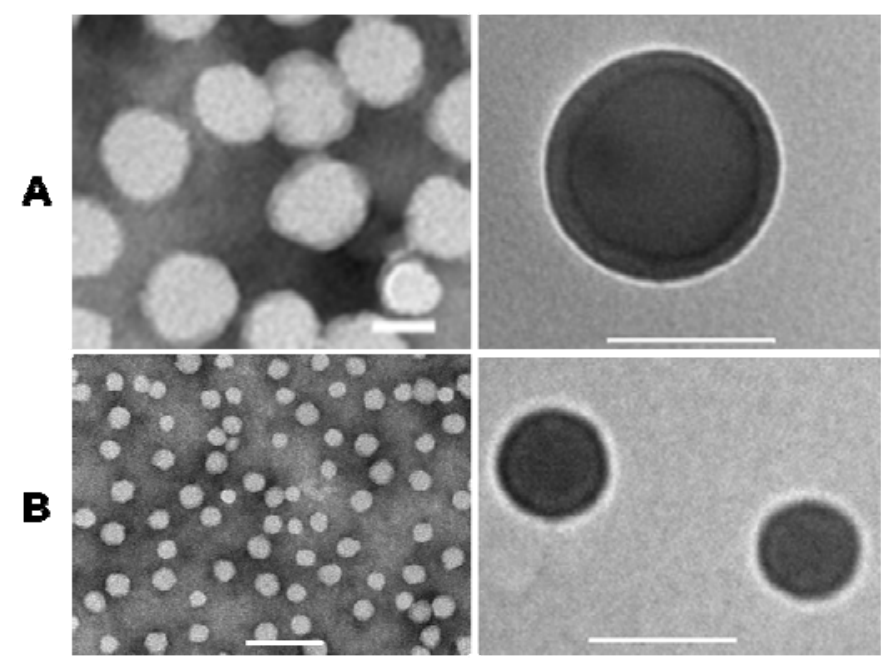

Figure 3: Transmission electronic microscopy of $R$-C nanoparticles (raw A) and R-CP nanoparticles (raw $B$ ). For observation at low magnification the nanoparticles were stained with phosphotungstic acid for $1 \mathrm{~min}$. Nanoparticles observed at high magnification were stained with phosphotungstic acid for $30 \mathrm{~s}$. Scale bar: $100 \mathrm{~nm}$

After lyophilization, all nanoparticles were totally soluble in DMSO while they were only partly soluble in THF and were insoluble in water and acidified water. This was in contrast with the solubility of chitosan, which was only soluble in acidified water, and with that of PIBCA which was fully soluble in THF. The difference in solubility shown between the nanoparticles and the homopolymers was a first indication that the nanoparticles were composed of amphiphilic copolymers including PIBCA for the hydrophobic part and chitosan and/or pluronic ${ }^{\circ} \mathrm{F} 68$ as the hydrophilic part.

In another experiment, small aliquots of water were added to R-CP and R-P nanoparticles which appeared almost completely soluble in THF. The addition of a total volume of $60 \mu \mathrm{l}(3 * 20 \mu \mathrm{L})$ of water to $300 \mu \mathrm{l}$ of THF containing $5 \mathrm{mg}$ of lyophilized nanoparticles led to a suspension of nanoparticles. In contrast, a precipitate appeared by repeating the same experiment with the homopolymer of PIBCA. Results from this experiment also suggested that R-CP and R-P nanoparticles were formed by copolymers including respectively PIBCA-chitosan-pluronic ${ }^{\circ} \mathrm{F} 68$ and PIBCA-pluronic ${ }^{\circ}$ F68.

\subsection{Composition in PIBCA, chitosan and pluronic 68 of the nanoparticles}

Before determining the composition of nanoparticles in PIBCA, chitosan and pluronic ${ }^{\circ} \mathrm{F} 68$, it was necessary to check that all the non associated chitosan and pluronic ${ }^{\circ} \mathrm{F} 68$ were removed from the nanoparticle suspensions during preparation. In case these conditions were not fulfilled, it was necessary to evaluate the remaining free fraction. No remaining chitosan could be detected after dialysis of a solution of chitosan with an initial concentration identical to that used in polymerization 
media. In contrast, by applying the same procedure with a solution of pluronic ${ }^{\circ} 68$, a significant fraction of the initial amount of pluronic ${ }^{\circ} \mathrm{F} 68$ dissolved in the solution $(15 \% \mathrm{w} / \mathrm{w})$ remained in the dialyzing bag. Results from this preliminary experiment showed that the composition of the nanoparticles in PIBCA and in chitosan can be determined directly on the suspension obtained from the dialysis when the nanoparticles were prepared in the absence of pluronic ${ }^{\circ} \mathrm{F} 68$. However, analysis of the composition of nanoparticles obtained in the presence of pluronic ${ }^{\circ}$ F68 required that the nanoparticles needed to be further purified and separated from the dispersing medium which contained a significant amount of remaining free pluronic ${ }^{\circ}$ F68 not eliminated by dialysis. These nanoparticles were then further purified by ultracentrifugation, washed with water and analyses were performed on the washed lyophilized nanoparticles isolated from the pellets.

Figure 4 shows the ${ }^{1} \mathrm{H}$ NMR spectra of pluronic ${ }^{\circ}$ F68 (Figure 4A), homoPIBCA (Figure 4B), chitosan (Figure $4 \mathrm{C}$ ) and of polymers soluble in DMSO composing the different nanoparticles (Figure 4D-G). The ${ }^{1} \mathrm{H}$ NMR spectrum from DMSO- $d_{6}$ solutions of lyophilized R-C nanoparticles revealed signals of PIBCA [ $\delta(\mathrm{ppm}): 3.92(\mathrm{~s}, 2 \mathrm{H}), 3.00-2.50(\mathrm{~s}, 2 \mathrm{H}), 1.96(\mathrm{~s}, 1 \mathrm{H}), 0.95(6 \mathrm{H})]$ (Figure 4F). The ${ }^{1} \mathrm{H}$ NMR spectrum from DMSO- $d_{6}$ solutions of lyophilized R-CP nanoparticles showed signals of PIBCA as mentioned above and signals of the pluronic ${ }^{\circ} 68[\delta(\mathrm{ppm}): 4.7(\mathrm{~s}, 2 \mathrm{H}), 3.6(\mathrm{~s}, 16 \mathrm{H}), 1,1(\mathrm{~d}, 2 \mathrm{H})]$ (Figure 4G). This clearly indicated that the nanoparticles were composed by both PIBCA and pluronic ${ }^{\circ}$ F68. Spectra recorded from DMSO- $d_{6}$ solutions of lyophilized A-C and A-CP nanoparticles were identical to those given by the corresponding nanoparticles prepared by method $\mathrm{R}$ (data not shown). These ${ }^{1} \mathrm{H}$ NMR does not exhibit signals of chitosan probably due to a too low concentration of chitosan in the nanoparticles and an overlapping with PIBCA signals.

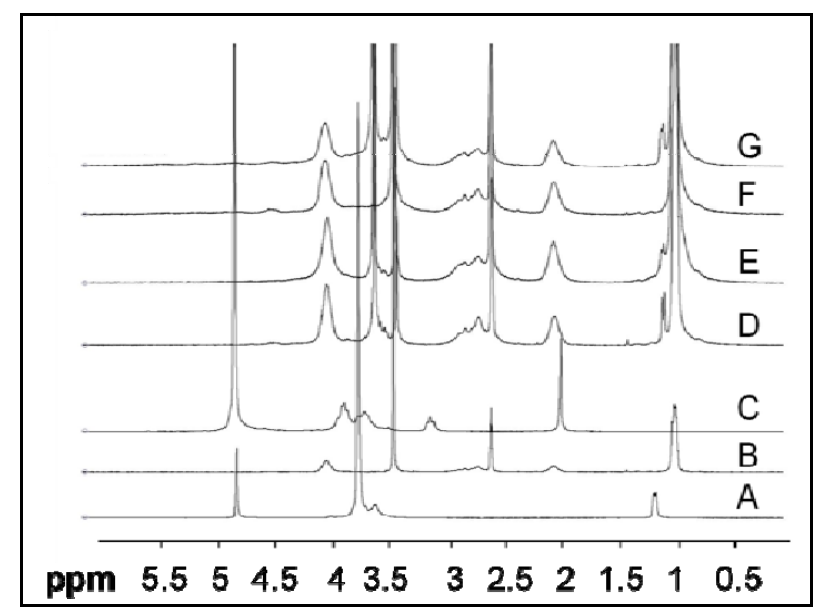

Figure 4: ${ }^{1} H$ NMR spectra of pluronic F68 (A); of PIBCA (B); of chitosan (C); $A-P$ nanoparticles (D); $R-P$ nanoparticles (E); $R$-C nanoparticles (F); $R$-CP nanoparticles (G).

Copolymerization between pluronic ${ }^{\circ} \mathrm{F} 68$ and IBCA was highlighted by the shift of the broad singlet at $4.5 \mathrm{ppm}$ corresponding to the hydroxyl function (Figure 4A). No difference was highlighted on ${ }^{1} \mathrm{H}$ NMR spectra for nanoparticles containing pluronic ${ }^{\circ} 68$ and IBCA prepared by method R or A. This suggested that, in both cases, the surroundings of hydroxyl functions of pluronic ${ }^{\circ} \mathrm{F} 68$ were involved in the formation of polymers composing nanoparticles (Figure 4).

The composition of nanoparticles determined by elemental analyses revealed a percentage in weight of IBCA around $80 \%$ (Table 2). This agreed quite well with the theoretical amount of IBCA introduced in the polymerization medium and indicated that the monomer was totally converted into polymer as it can be expected with such a highly reactive species. Percentages of pluronic ${ }^{\circ}$ F68 found associated with nanoparticles R-CP and A-CP were 15 and $24 \%$ respectively. Those associated with nanoparticles prepared with pluronic were 27 and $30 \%$ for the R-P and A-P nanoparticles respectively. The content of the nanoparticles in pluronic ${ }^{\circ} \mathrm{F} 68$ was greatly influenced by the method of polymerization when polymerizations were performed in the presence of both chitosan and pluronic ${ }^{\circ}$ F68. In contrast, it was close to $30 \%$ independently of the methods of polymerization when 
the nanoparticles were prepared with pluronic ${ }^{\circ} \mathrm{F} 68$ as sole hydrophilic polymer introduced in the polymerization medium. It is noteworthy that this result agreed with the composition in PIBCA found for these nanoparticles.

Table 2. Percentage in weight of PIBCA in the preparation of nanoparticles as deduced from the different experiments

\begin{tabular}{c|c|c|c}
\hline & R-CP & R-C & R-P \\
\hline Elemental analysis & 85 & 80 & 73 \\
Basic hydrolysis (NaOH) & 80 & 60 & 79 \\
Basic hydrolysis (NaH) & 80 & 77 & $/$ \\
Acid hydrolysis & 72 & 83 & 78 \\
\hline & A-CP & A-C & A-P \\
\hline Elemental analysis & 68 & 89 & 70 \\
Basic hydrolysis (NaOH) & $52^{\text {a }}$ & $/$ & 83 \\
Basic hydrolysis (NaH) & $/$ & $/$ & $/$ \\
Acid hydrolysis & 63 & 74 & 73 \\
\hline
\end{tabular}

For all samples, the amount of PIBCA expected from the experimental procedures (methods $\mathrm{R}$ and A) was $77 \%$.

${ }^{a}$ Yield without SPE filtration (see methods)

\subsection{Characteristics of polymers forming the nanoparticles}

The characteristics of polymers forming the nanoparticles were achieved from different fractions. One fraction corresponded to polymers soluble in THF collected after dissolution of nanoparticles in THF. Others were obtained in this solvent and after treatment in different conditions used to destroy selectively either the PIBCA or the chitosan part of polymers composing the nanoparticles. A summary of the scheme followed for this characterization is given in the Figure 2.

\subsubsection{Analysis of the fraction of polymers soluble in THF}

As shown in Table 3, all the nanoparticles containing both chitosan and pluronic ${ }^{\circ} \mathrm{F} 68$ dissolved much better in THF than those prepared with chitosan only. Indeed, the solubility in THF of nanoparticles containing chitosan and pluronic ${ }^{\circ} \mathrm{F} 68$ was $91 \%$, while R-P and A-P nanoparticles dissolved totally in THF. This can be explained by the fact that pluronic ${ }^{\circ} \mathrm{F} 68$ is soluble in THF while chitosan is not. The SEC chromatogram of the THF-soluble polymers obtained from R-C nanoparticles showed two peaks, with elution time at 13 and $22 \mathrm{~min}$ with an average molecular weight of $48000 \mathrm{~g} / \mathrm{mol}$ (Figure 5B). The presence of two peaks on the SEC chromatograms can be explained by a competition between two mechanisms of polymerization. This was also suggested by the high polydispersity index of 15.8 for THF-soluble polymers recovered from R-C nanoparticles (Table 3 ). THF-soluble polymers recovered from R-CP nanoparticles were given a chromatogram with a very broad peak with a maximum at $16 \mathrm{~min}$ and a clear shoulder shown at elution time ranging from 18 and $21 \mathrm{~min}$ (Figure $5 A)$. This also can be explained by a competition between two mechanisms of polymerization in which the balance between the two mechanisms of polymerization was different compared to the previous case. As expected from the profiles of the SEC chromatogram, polydispersity index was also much lower for R-CP nanoparticles compared with that of R-C nanoparticles (Table 3). SEC chromatograms of the THF-soluble polymers obtained from A-CP and A-C nanoparticles revealed a single peak with an elution time at respectively 19 and 20 min (Figures 5D and 5E). The corresponding molecular weights were respectively 7590 and $5530 \mathrm{~g} / \mathrm{mol}$. THF-soluble polymers recovered from R-P nanoparticles showed a very broad peak with a maximum at $18 \mathrm{~min}$ and a clear shoulder shown at elution time ranging from 13 and $17 \mathrm{~min}$ (Figure $5 \mathrm{C}$ ). This can be explained by a competition between radical and anionic polymerization mechanisms with a prevalence of the anionic initiation. THF-soluble polymers recovered from A-P nanoparticles showed a chromatogram similar to the one obtained for A-CP nanoparticles (Figure 5F).

As expected from the profiles of the SEC chromatograms, polydispersity index was much lower for nanoparticles prepared by method $A$ than those prepared by method $R$. 
Table 3. Results from the SEC analysis of THF soluble part of the polymers and of the polymers recovered after acid hydrolysis of the nanoparticles.

\begin{tabular}{c|c|c|c|c}
\hline \multirow{2}{*}{} & $\begin{array}{c}\text { Fraction } \\
\text { soluble } \\
\text { in THF }\end{array}$ & Mw global & Mw1* & Pi \\
\cline { 2 - 3 } \% & \multicolumn{2}{|c|}{ g/mol } \\
\hline \hline \multicolumn{4}{c}{ THF soluble fraction } \\
R-CP & 91 & 86470 & 140330 & 2.5 \\
R-C & 57 & 48000 & 157380 & 15.8 \\
R-P & 100 & 44510 & 285330 & 3.0 \\
A-CP & 100 & 7590 & $/$ & 2.1 \\
A-C & 80 & 5530 & $/$ & 2.8 \\
A-P & 100 & 7100 & $/$ & 1.2 \\
\hline \hline \multicolumn{5}{c}{ Polymer recovered after acid hydrolysis } \\
\hline \hline R-CP & 100 & 100620 & 154450 & 2.5 \\
R-C & 100 & 77440 & 294820 & 21.1 \\
R-P & 100 & 62700 & 192390 & 3.7 \\
A-CP & 100 & 6270 & $/$ & 1.4 \\
A-C & 100 & 5070 & $/$ & 1.9 \\
A-P & 100 & 6230 & $/$ & 1.5 \\
\hline
\end{tabular}

* Mw1 correspond to the peak eluting between 13 and $17.5 \pm$ 0.5 min corresponding to radical polymerization
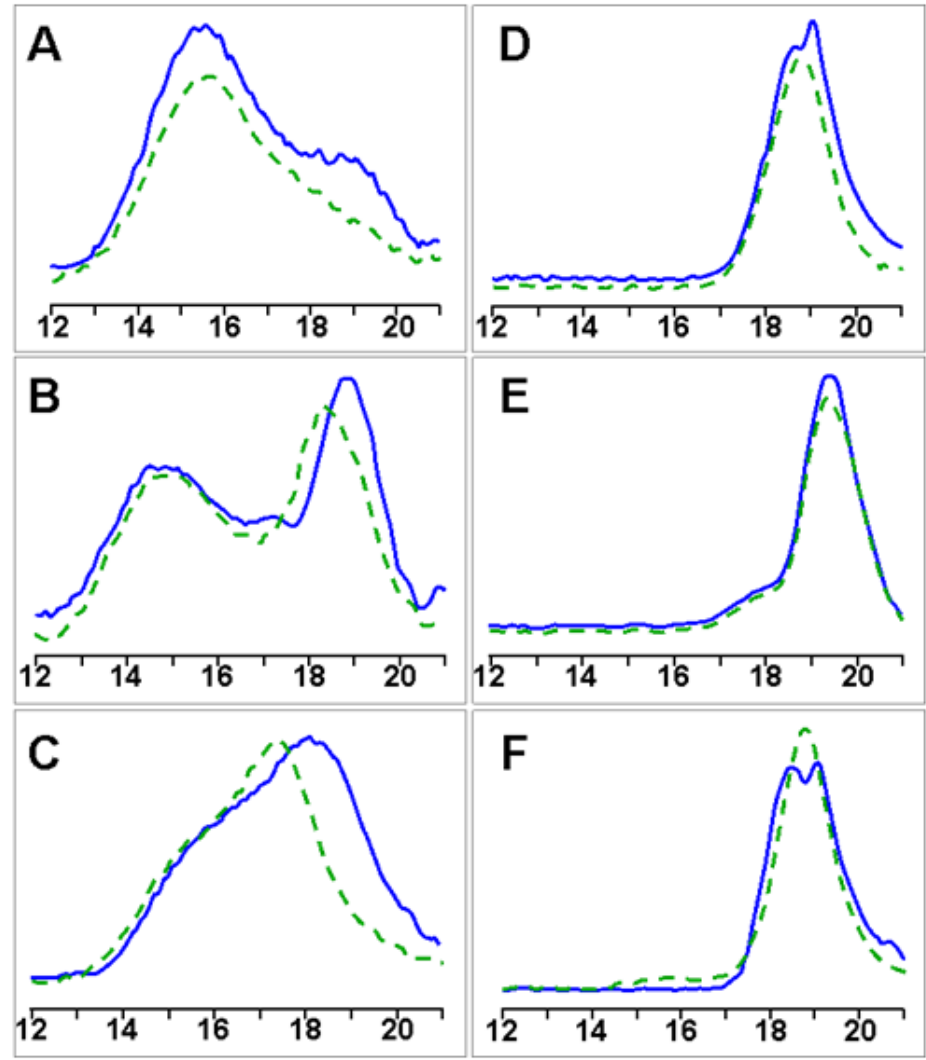

Figure 5: Chromatograms given by SEC profile given by the analysis of the THF soluble polymer composing nanoparticles (solid line) and of polymer recovered after acid hydrolysis of the nanoparticles (dotted line). A: $R$-CP nanoparticles, $B: R$-C nanoparticles, $C: R-P$ nanoparticles, $D: A-C P$ nanoparticles, E: A-C nanoparticles, F: A-P nanoparticles. 


\subsubsection{Analysis of polymers recovered from acid hydrolysis of the nanoparticles}

The chemical structure of the residues obtained after acid hydrolysis were determined by ${ }^{1} \mathrm{H}$ NMR. Spectra obtained from the analysis of residues of nanoparticles prepared by method $\mathrm{R}$ are reported in Figure 6. Spectra recorded from the analysis of residues of nanoparticles prepared by method $A$ were very similar (data not shown). After acid hydrolysis, residues were totally soluble in THF and signals showed on ${ }^{1} \mathrm{H}$ NMR spectra corresponded to PIBCA [ $\delta(\mathrm{ppm}): 3.92(\mathrm{~s}, 2 \mathrm{H}), 3.00-2.50(\mathrm{~s}, 2 \mathrm{H})$, $1.96(\mathrm{~s}, 1 \mathrm{H}), 0.95(6 \mathrm{H})]$.

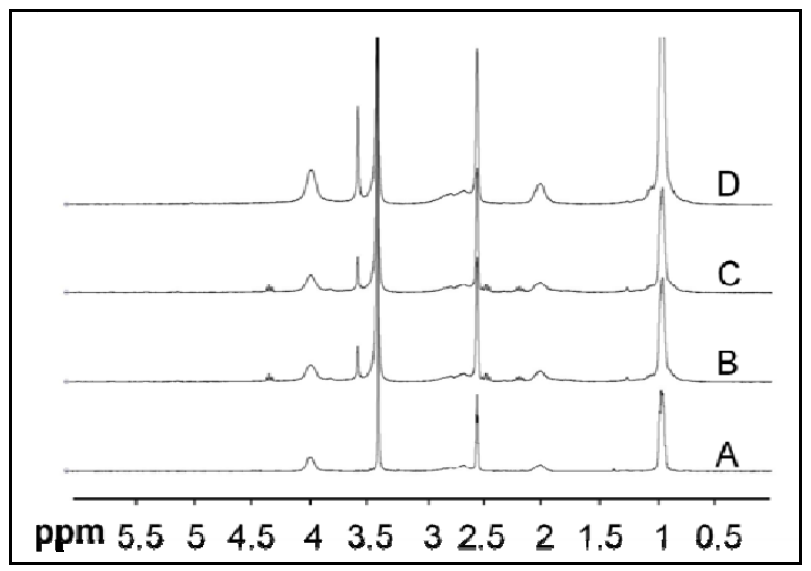

Figure 6: ${ }^{1} H$ NMR spectra of PIBCA (A) and after acid hydrolysis of $R$-CP nanoparticles (B); $R-C$ nanoparticles $(C)$ and $R-P$ nanoparticles $(D)$.

For most samples, the percentage in weight of polymer recovered after acid hydrolysis ranged between 73 and $83 \%$. It was the expected amount calculated from the amount of PIBCA introduced in the polymerization medium (Table 2 ) except for the polymer recovered from acid hydrolysis of ACP nanoparticles (63\%).

For nanoparticles prepared by method R, SEC chromatograms of polymer recovered after acid hydrolysis showed either two peaks or a broad asymmetric peak corresponding to polymers with average molecular weight ranging from 62700 to $100620 \mathrm{~g} / \mathrm{mol}$ (Figure 6, Table 3). This was also in favor with the hypothesis that there was a competition between two mechanisms of polymerization when nanoparticles were prepared by the method $R$. The difference between the chromatograms can be explained by the balance in the competition between the two mechanisms of polymerization.

On the contrary, for nanoparticles prepared by method A, SEC chromatograms of polymer recovered after acid hydrolysis showed only one peak with an elution time at $19.5 \pm 0.5 \mathrm{~min}$ (Figure 6). The molecular weight of the polymer ranged from 5070 and $6230 \mathrm{~g} / \mathrm{mol}$ (Table 3).

SEC chromatograms of polymer recovered from acid hydrolysis of R-P nanoparticles showed also a broad peak with a maximum at $18 \mathrm{~min}$ and a shoulder at elution time ranging from 13 to $17 \mathrm{~min}$. This indicated the occurrence of a competition between the two mechanisms of polymerization. On the contrary, SEC chromatogram of polymer recovered from acid hydrolysis of A-P nanoparticles showed only one peak at elution time $19 \mathrm{~min}$ in agreement with a single polymerization mechanism.

As expected from the profiles of the SEC chromatograms, polydispersity index was also much lower for nanoparticles prepared by method A than those of nanoparticles prepared by method R (Table 3 ).

\subsubsection{Analysis of polymers recovered from basic hydrolysis of the nanoparticles}

The ${ }^{1} \mathrm{H}$ NMR spectrum of the yellow solid recovered from basic hydrolysis with $\mathrm{NaOH}$ of $\mathrm{R}-\mathrm{C}$ and $\mathrm{R}-\mathrm{CP}$ nanoparticles showed a broad signal between 2 and 3 ppm (Figure 7). These signals can be attributed to the presence of cyanopropionic acid residues in the structure of the analyzed compound. Intensity of the signals was higher for compounds recovered from basic hydrolysis of R-CP and of R-C nanoparticles than that obtained from compounds recovered from the basic hydrolysis of R-P and of A-P nanoparticles. It suggested that IBCA linked to pluronic ${ }^{\circ} \mathrm{F} 68$ either by anionic or by radical mechanism was totally hydrolyzed with $\mathrm{NaOH}$. In contrast, the hydrolysis was only partial considering 
R-CP and R-C nanoparticles suggesting that linkages between PIBCA and chitosan which were not hydrolyzed by $\mathrm{NaOH}$.

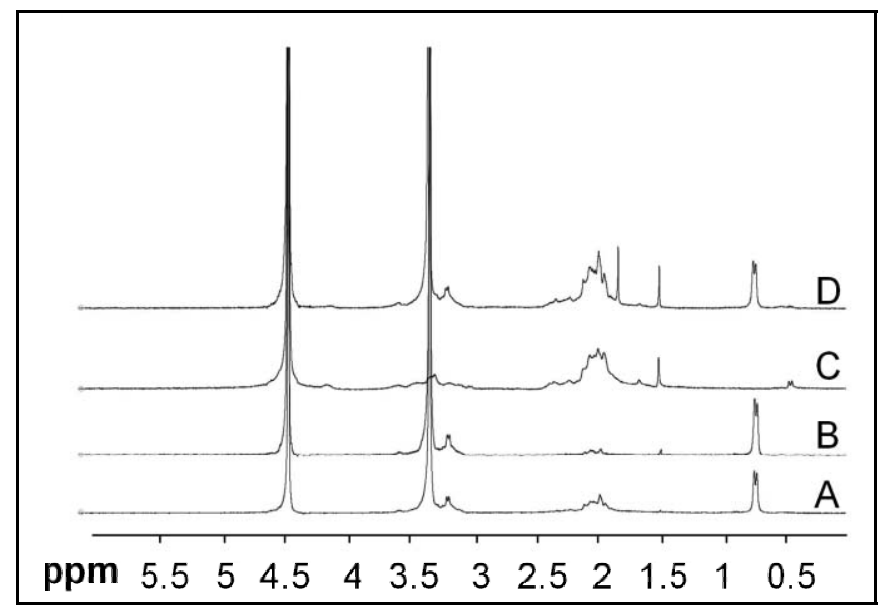

Figure 7: ${ }^{1} H$ NMR after basic hydrolysis with $N a O H$ of $R$ - $P$ nanoparticles $(A)$; of $A-P$ nanoparticles $(B)$ of $R$ $C$ nanoparticles $(C)$ and of $R$-CP nanoparticles (D).

To further characterize the structure of the residue recovered after basic hydrolysis with $\mathrm{NaOH}$ of $\mathrm{R}-\mathrm{C}$ and R-CP nanoparticles, a filtration on SPE cartridge was performed. This contributed to the disappearance of the broad signal shown between 2 and 3 ppm for the R-CP nanoparticles suggesting that the PIBCA linked to chitosan was totally removed after basic hydrolysis (Figures $8 \mathrm{~A}$ and B). In contrast, this signal remained in the spectra obtained after SPE filtration from the residue recovered after hydrolysis of the R-C nanoparticles (Figure $8 \mathrm{C}$ and $\mathrm{D}$ ). The percentage of hydrophilic polymer, i.e. chitosan/pluronic ${ }^{\circ}$ F6, in the R-CP nanoparticles was $20 \%$ as determined by gravimetry. All these results clearly showed that the structures of the copolymers forming R-CP and R-C nanoparticles were different.

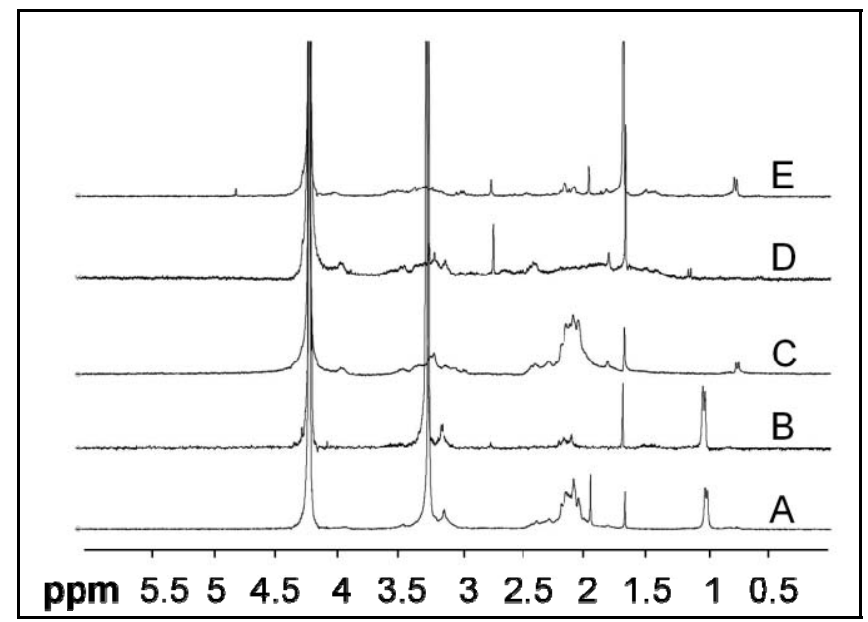

Figure 8: ${ }^{1} H$ NMR spectra after hydrolysis with $\mathrm{NaOH}$ of $\mathrm{R}$-CP nanoparticles (A), hydrolysis with $\mathrm{NaOH}$ and SPE filtration of R-PC nanoparticles (B), hydrolysis with NaOH of $R$-C nanoparticles (C), hydrolysis with $\mathrm{NaOH}$ and SPE filtration of $R-\mathrm{C}$ nanoparticles $(D)$, after hydrolysis with NaH and SPE filtration of R-C nanoparticles (E). 
To obtain full hydrolysis of PIBCA part of the polymers composing the nanoparticle, R-C nanoparticles were hydrolyzed with $\mathrm{NaH}$ in THF. The figure $8 \mathrm{E}$ showed the ${ }^{1} \mathrm{H}$ NMR spectra of the residue recovered after hydrolysis and filtration on SEP cartridge. Signals expected from PIBCA ( 2 and 3 ppm) were almost vanished indicating that this method of hydrolysis was suitable to remove IBCA residues. This made possible the determination of the percentage $(23 \%)$ of chitosan composing the R-C nanoparticles (Table 4). The ${ }^{1} \mathrm{H}$ NMR spectrum of the slightly yellow solid residue obtained from treatment after hydrolysis with $\mathrm{NaH}$ of R-CP nanoparticles and SPE filtration also showed the disappearance of the broad signal between 2 and 3 ppm (data not shown).

Table 4. Percentage in weight of chitosan determined form elemental analysis of the nanoparticles and recovered after basic hydrolysis performed in different conditions

\begin{tabular}{c|c|c}
\hline$\%(w / w)$ & R-CP & R-C \\
\hline $\begin{array}{c}\text { Elemental analyses of } \\
\text { nanoparticules }\end{array}$ & 6 & 20 \\
\hline $\begin{array}{c}\text { Residue after basic hydrolysis } \\
\text { (NaOH), SPE filtration and } \\
\text { elemental analysis }\end{array}$ & 6 & nd \\
\hline $\begin{array}{c}\text { Residue after basic hydrolysis } \\
\text { (NaH), SPE filtration and } \\
\text { elemental analysis }\end{array}$ & 4 & 23 \\
\hline
\end{tabular}

By elemental analysis of nitrogen on the residue recovered after hydrolysis by $\mathrm{NaOH}$ or $\mathrm{NaH}$ of $\mathrm{R}-\mathrm{CP}$ nanoparticles which included both pluronic ${ }^{\circ} \mathrm{F} 68$ and chitosan, it was possible to evaluate the composition in chitosan of these nanoparticles. It could be found that chitosan represented between 4 and $6 \%$ of the total amount of polymer composing these nanoparticles (Table 4).

\subsubsection{Determination of the free amine functions}

The percentage of free amine functions on nanoparticles was determined as an indicator of the balance between the anionic and radical polymerization initiation mechanism. As expected from a control experiment, R-P nanoparticles does not reveal any coloration with ninhydrin reagent because none of pluronic ${ }^{\circledR} \mathrm{F} 68$ and PIBCA contained free amino group in their structure. The amount of free amine functions was higher on R-CP nanoparticles than on A-CP nanoparticles and agreed with the respective mechanism of polymerization (Table 5). This confirmed that the ratio between the two polymerization mechanisms was in favor to the radical polymerization during the preparation of the $\mathrm{R}-\mathrm{CP}$ nanoparticles. In nanoparticles prepared in the absence of pluronic ${ }^{\circ} \mathrm{F} 68$, the number of free amine functions was identical.

Table 5. Amount of free amine functions found on nanoparticles ( $\mathrm{nmol} / \mathrm{mg}$ of polymer)

\begin{tabular}{c|c|c|c}
\hline & $\begin{array}{c}\text { Concentration } \\
\text { of } \\
\text { nanoparticles } \\
\text { (mg/mL) }\end{array}$ & $\begin{array}{c}\text { Free amine } \\
\text { functions } \\
\text { (nmol/mg } \\
\text { of polymer) }\end{array}$ & $\begin{array}{c}\text { Free amine } \\
\text { functions } \\
\text { (nmol/mg } \\
\text { of chitosan) }\end{array}$ \\
\hline R-CP & 35 & 5.4 & 90 \\
\hline R-C & 31 & 2.2 & 11 \\
\hline A-CP & 24 & 2.9 & 29 \\
\hline A-C & 44 & 1.4 & 12.7 \\
\hline
\end{tabular}

\subsection{Evaluation of the capacity of the nanoparticles to activate the complement system}

In absence of pluronic ${ }^{\circ}$ F68 and independently of the method of polymerization, the complement system was frankly activated during the incubation of the nanoparticles with serum. This was clearly showed by the dramatic increase of the peak corresponding to C3b on the electrophoregrams (peak shown on the right side). The complement activation factor was found above $50 \%$ for both R-C and 
A-C nanoparticles (Table 6). The nanoparticles prepared with pluronic ${ }^{\circ} \mathrm{F} 68$ showed a lower capacity to activate the complement system with a complement activation factor lower than $20 \%$ (Table 6).

Table 6: Complement activation profile and Complement activation factor (CAF) given by the different types of nanoparticles. Structures of copolymers composing the nanoparticles and models suggested for the arrangement of the hydrophilic chains at the nanoparticle surface (red threads: chitosan, blue threads: pluronic $\left.{ }^{\circ} 68\right)$

\begin{tabular}{|c|c|c|c|c|}
\hline Nanoparticles & $\begin{array}{c}\text { Complement activation } \\
\text { (2D } \\
\text { immunoelectrophoregram) }\end{array}$ & $\begin{array}{l}\text { CAF } \\
(\%)\end{array}$ & $\begin{array}{l}\text { Structures of } \\
\text { copolymers* }\end{array}$ & $\begin{array}{c}\text { Model of the } \\
\text { arrangement } \\
\text { of } \\
\text { hydrophilic } \\
\text { chains at the } \\
\text { nanoparticle } \\
\text { surface }\end{array}$ \\
\hline R-CP & & 20 & $\begin{array}{l}1 . \mathrm{I} \\
2 . \mathrm{I} \\
2 . \mathrm{II} \\
2 . \mathrm{III} \\
2 . \mathrm{IV}\end{array}$ & \\
\hline R-C & & 66 & $\begin{array}{l}1 . \mathrm{I} \\
1 . \mathrm{II} \\
1 . \mathrm{III}\end{array}$ & \\
\hline R-P & & 20 & $\begin{array}{l}2 . \mathrm{I} \\
2 . \mathrm{II} \\
2 . \mathrm{III} \\
2 . \mathrm{IV}\end{array}$ & \\
\hline $\mathbf{A - C P}$ & & 14 & 1.II & \\
\hline A-C & & 70 & $\begin{array}{l}1 . \mathrm{II} \\
2 . \mathrm{II} \\
2 . \mathrm{III}\end{array}$ & \\
\hline A-P & & 16 & $\begin{array}{l}2 . \mathrm{I} \\
2 . \mathrm{II} \\
2 . \mathrm{III} \\
2 . \mathrm{IV}\end{array}$ & 纪 \\
\hline
\end{tabular}

*scheme of the structures given in figures 1 


\section{Discussion:}

Media used for the preparation of PIBCA nanoparticles designed for in vivo delivery of siRNA contain pluronic ${ }^{\circ} \mathrm{F} 68$ and chitosan. ${ }^{19}$ The general anionic polymerizations of IBCA performed in those media can be rather complex including competitions during the initiation stage of the polymerization. In the conditions of radical polymerization given by the presence of $\mathrm{Ce}^{4+}$ in the polymerization medium, the competition between sites of polymerization initiation may be even more complicated as both pluronic ${ }^{\circ} \mathrm{F} 68$ and chitosan can initiate both radical and anionic polymerizations. This can influence greatly the structure of polymers composing the nanoparticles and in turn the in vivo fate of the drug carrier. Characterizations performed in this work was aimed to elucidate the structure of the polymers forming the nanoparticles and obtained in the different conditions of polymerizations. From these structures, it was aimed to draw models of the conformation of the chains of the hydrophilic polymers stranded at the nanoparticle surface and to investigate whether a correlation could be done with the capacity of the nanoparticles to trigger the activation of the complement system.

The hypothesis of a competition between radical and anionic initiation of the polymerization during nanoparticles preparation was confirmed by SEC analysis performed either on the part of nanoparticles R-CP or R-C soluble in THF or on the PIBCA recovered from acid hydrolysis of the same nanoparticles. The first peak eluting at $16 \mathrm{~min}$ corresponded to polymers formed by radical polymerization because it only appeared on nanoparticles prepared by method $\mathrm{R} .{ }^{20}$ The second peak eluting at $19.5 \pm 0.5 \mathrm{~min}$ corresponded to polymer formed by anionic polymerization. This is the only peak appearing from the analysis of the polymer of nanoparticles prepared by method $A$ and it was also shown on several nanoparticles prepared by method R. Regarding the balance between the two peaks shown on the chromatograms, it can be concluded that anionic polymerization was less important during the synthesis of the R-CP nanoparticles than that of the R-C nanoparticles.

The ratio between radical and anionic polymerization for $\mathrm{R}-\mathrm{CP}$ and $\mathrm{R}-\mathrm{C}$ polymerization conditions can be deduced from SEC analysis of the polymers obtained after acid hydrolysis (Table 3 ) by using the following equations:

$$
\begin{gathered}
M_{\mathrm{w}(E-C D)}=a \times M_{\mathrm{w} 1}(E-C D)+b \times M_{\mathrm{w} 2(\Delta-C D)} \\
a+b=1
\end{gathered}
$$

where $a$ is the fraction of radical polymerization and $b$ the fraction of anionic polymerization.

$\mathrm{Mw}$ is the global molecular weight evaluated on average peak eluting from 13 to $21 \mathrm{~min} ; \mathrm{M}_{\mathrm{w} 1}$ was evaluated directly from SEC chromatograms of polymers recovered from acid hydrolysis of R-CP, R-C and R-P nanoparticles (peak eluting from 13 to $18 \mathrm{~min}$ ) and $\mathrm{Mw} 2$ was approximated as the molecular weight of the polymers recovered after acid hydrolysis of A-CP, A-C and A-P nanoparticles respectively. It was also assumed that polymer with $M_{w 1}$ resulted from the radical polymerization whereas polymer with $M_{w 2}$ resulted from the anionic polymerization. This assumption seemed reasonable because only polymer with $M_{w 2}$ appeared on chromatograms obtained from the analysis of nanoparticles prepared by method $A$.

The presence of pluronic ${ }^{\circ} \mathrm{F} 68$ in polymerization medium for the preparation of R-CP nanoparticles modified the ratio of radical/anionic polymerization in favor to the radical polymerization (Table 7). This might be due to hydrogen bonds between the hydroxyl groups of pluronic ${ }^{\circ} \mathrm{F} 68$ and the free amine of chitosan. Concerning the R-P nanoparticles, the radical initiation mediated by $\mathrm{Ce}^{4+}$ ions on PEG chain was slow due to the formation of only one radical per PEG chains. ${ }^{30}$ In agreement with these results, the ratio radical/anionic polymerization was in favor of anionic polymerization in these conditions of polymerization (Table 7).

The composition of nanoparticles was also affected by the presence of pluronic ${ }^{\circ} \mathrm{F} 68$. Whereas R-C nanoparticles were composed of around $20 \%$ of chitosan, in agreement with our previous study considering the preparation of PIBCA nanoparticles with another polysaccharide. ${ }^{20}$ The R-CP nanoparticles were composed of 5 to $6 \%$ of chitosan and $15 \%$ of pluronic ${ }^{\circ}$ F68. 
Table 7. Ratio between radical and anionic polymerization in $R-C P$ and $R-C$ nanoparticles and distribution of the Mw of PIBCA part of polymers composing the nanoparticles.

\begin{tabular}{c|c|c|c|c} 
& Mw & Mw1 & Mw2 & \\
\hline & \multicolumn{3}{|c|}{ g/mol } & Radical/anionic \\
\hline R-CP & 100620 & 154450 & 6270 & $64 / 36$ \\
\hline R-C & 77440 & 294820 & 5070 & $25 / 75$ \\
\hline R-P & 62700 & 192390 & 6230 & $30 / 70$ \\
\hline
\end{tabular}

Structures of copolymers composing nanoparticles could be deduced from the results provided by the basic hydrolysis experiments. Sodium hydroxide was able to hydrolyze all PIBCA in the case of RCP nanoparticles confirming that practically no IBCA was polymerized by the anionic mechanism initiated by chitosan. The anionic polymerization only occurred by an initiation on pluronic ${ }^{\circ} \mathrm{F} 68$. Thus according to the hypothesis, the R-CP nanoparticles were formed by linear copolymers of chitosanPIBCA (structure 1.I,1.II, 1.III on Figure 1) and by linear and branched copolymers of pluronic ${ }^{\circ} \mathrm{F} 68$ PIBCA (structure 2.I, 2.II, 2.III, 2.IV on Figure 1).

Partial hydrolysis of PIBCA obtained with $\mathrm{NaOH}$ for $\mathrm{R}-\mathrm{C}$ nanoparticles indicated that they were composed of polymers of different structures compared to those found in R-CP nanoparticles. A polymerization initiation through an anionic mechanism on the primary amine function of chitosan can explain the difference. The need of a reducing agent to remove all PIBCA residues on R-C nanoparticles confirmed the presence of $\mathrm{C}-\mathrm{N}$ linkage due to the initiation of the anionic polymerization on chitosan. Results from the dosage of the amine functions also agreed with this conclusion considering that the content in free amines was more important in the R-CP nanoparticles than in the R-C nanoparticles. It can be concluded that the R-C nanoparticles were formed by branched and linear copolymers of chitosan (structure 1.I, 1.II and 1.III on Figure 1). Structures of copolymers composing the other types of nanoparticles were conformed to those expected from the hypothesis (Table 6)

From the results of the analysis of the nanoparticles, it can be concluded that the organization of the hydrophilic chains at the surface of the nanoparticles depended on both the method of polymerization and the presence or not of pluronic ${ }^{\circ} \mathrm{F} 68$. The surface of R-CP nanoparticles was composed in a majority of linear brush of pluronic ${ }^{\circ} \mathrm{F} 68$ and chitosan (Table 6). On the contrary, the surface of R-C nanoparticles appeared to be mainly composed of loops of chitosan chains with a few chains on the brush conformation. The surface of R-P nanoparticles was composed of brush and loops of pluronic ${ }^{\circ} \mathrm{F} 68$ chains. A majority of these chains may take a conformation of brush due to the predominance of the formation of only one radical per polymer chain and also only one initiation by the hydroxyl function by the anionic mechanism. Nanoparticles prepared by method A in absence of pluronic ${ }^{\circ} \mathrm{F} 68$ have polymers chains in the loop conformation on their surface whereas in presence of pluronic ${ }^{\circ} \mathrm{F} 68$, the surface of the nanoparticles is composed of loops of chitosan and pluronic ${ }^{\circ} \mathrm{F} 68$ and also of linear brush of pluronic ${ }^{\circ}$ F68 (Table 6).

These structures deduced from all the above results agreed quite well with the complement activation profile shown by the different types of nanoparticles. Indeed, all nanoparticles that showed hydrophilic chains with a brush conformation did not activate the complement system. In contrast, the nanoparticles $\mathrm{R}-\mathrm{C}$ and $\mathrm{A}-\mathrm{C}$ on which the conformation of the chitosan chains was essentially formed by loops with eventually short chains in the brush configuration in the R-C nanoparticles were clearly activated the complement system. These results agreed well with recent results showing that the brush configuration of high density is highly efficient to hamper large proteins like C3 to reach the nanoparticle surface reducing the chance to trigger the activation of the complement system. ${ }^{10,17}$ In contrast, on the surface of nanoparticles protected with a polysaccharide in the loop conformation there are plenty of free space available for protein adsorption. This configuration is therefore more favorable to make possible activation of the complement system. ${ }^{10,17}$ Results from the complement activation of the R-CP nanoparticles found in this work provided some 
highlights to explain the remarkable efficacy of these nanoparticles where they were used to deliver a siRNA to a tumor after an intravenous administration. ${ }^{19}$ Their low capacity to activate the complement system agreed with their properties to escape uptake by defense systems of the organism making possible their distribution in tumors through the blood circulation and thanks to the enhanced permeation and retention (EPR) effect.

\section{Conclusion}

This work based on the analysis of the composition and structure of polymers composing the nanoparticles prepared by emulsion polymerization of IBCA have highlighted that anionic and radical polymerization coexisted when nanoparticles were prepared by the method $R$ in presence of chitosan. The balance between the two polymerization mechanisms was greatly modified by adding pluronic ${ }^{\circ} 68$ in the polymerization medium reducing the size of the nanoparticles. The nanoparticles were composed of copolymers which structures depending on the mechanism of polymerization and the hydrophilic polymer responsible for the polymerization initiation. The structure of these copolymers was elucidated. They were used to propose models for the conformational arrangement of the hydrophilic chains stranded at the surface of nanoparticles obtained in the different conditions of polymerization used in this work. The capacity of the nanoparticles to activate the complement was correlated with the proposed models of the nanoparticle surface structure. Thus, these results will be useful to optimize the synthesis of nanomaterials with low capacity to trigger the activation of the complement system for in vivo applications and for siRNA delivery.

\section{Acknowledgments}

The authors want to thank Karine Leblanc from Biocis (Chatenay Malabry, France) for the elemental analyses, Brit Nacke Erasmus student from Germany for the experiments of complement activation and Dr Henry De Martimprey for transmission electronic microscopy. This work was supported by InNaBioSanté project "NANOINTERFERENCE".

\section{References}

1. C. Vauthier, P. Couvreur, J Biomed. Nanotechnol., 3, 223 (2007)

2. S.M. Moghimi, A.C. Hunter, J.C. Murray FASEB Journal 19, 311 (2005)

3. G. Sharma, D.T. Valenta, Y. Altman, S. Harvey, H. Xie, S. Mitragotri, J.W. Smith, J. Control. Release 147,408 (2010)

4. T. Maldiney, C. Richard, J. Seguin, N. Wattier, M. Bessodes, D. Scherman, ACS Nano, 5, 854, (2011)

5. J. Szebeni, F. Muggia, A. Gabizon, Y.Barenholz, Adv Drug Del. Rev. 63, 1020 (2011)

6. K.N. Ekdahl, J.D. Lambris, H. Elwing, D. Ricklin, P.H. Nilsson, Y. Teramura, I.A. Nicholls, B. Nilsson Adv Drug Del. Rev. 63, 1042 (2011)

7. S.M. Moghimi, A.J. Andersen, D. Ahmadvand, P.P. Wibroe, T.L. Andresen, A.C. Hunter, Adv Drug Del. Rev. 63, 1000 (2011)

8. D.F. Moyano, V.M. Rotello, Langmuir 27, 10376 (2011)

9. C. Vauthier, P. Lindner, B. Cabane, Colloids Surf., B, 69, 207 (2009)

10. C. Vauthier, B. Persson, P. Lindner, B. Cabane, Biomaterials 32, 1646 (2011)

11. C.D. Walkey, J.B. Olsen, H. Guo, A. Emili, W.C.W. Chan, J. Am. Chem. Soc. (2011) doi 10.1021/ja2084338

12. K. Avgoustakis, Curr. Drug. Deliv., 1, 321 (2004)

13. D.E. Owens, N.A. Peppas, Int. J. Pharm., 307, 93 (2006)

14. C. Lemarchand, R. Gref, C. Passirani, E. Garcion, B. Petri, R. Müller, D. Costantini, P. Couvreur, Biomaterials 27, 108 (2006)

15. C. Passirani, G. Barratt, J.P. Devissaguet, D. Labarre, Pharm. Res. 15, 1046 (1998)

16. C. Chauvierre, D. Labarre, P. Couvreur, C. Vauthier, Macromolecules 36, 6018 (2003)

17. I. Bertholon, C. Vauthier, D. Labarre Pharm. Res. 23,1313 (2006)

18. A. Maksimenko, V. Polard, M. Villemeur, H. Elhamess, P. Couvreur, J.R. Bertrand, M. Aboubakar, M. Gottikh, C. Malvy, Ann. N Y Acad. Sci., 1058, 52 (2005) 
19. H. De Martimprey, J.R. Bertrand, C. Malvy, P. Couvreur, C. Vauthier, Pharm. Res. 27, 498 (2010) 20. I. Berthelot, S. Lesieur, D. Labarre, M. Besnard, C. Vauthier, Macromolecules 39, 3559 (2006) 21. M. Huang, E. Khor, L.Y. Lim, In Winter symposium \& $11^{\text {th }}$ international symposium on recent advances in drugs delivery systems, USA (2003)

22. T. Khan, K. Peh, H. Ch'ng, J. Pharm. Pharm. Sci., 3, 303 (2000)

23. Y. Liu, F. Tian, K.A. Hu, Carbohydr. Res. 339, 845 (2004)

24. E. Curotto, F. Aros, Ann. Biochem., 211, 240 (1993)

25. L. Liu, X. Xu, S. Guo, W. Han Carbohydr. Polym. 75, 401 (2009)

26. M.D. Kazatchkine, G. Hauptmann, Nydegger in Techniques du complement, Paris: INSERM (1986) 27. M. Vittaz, D. Bazile, G. Spenlehauer, T. Verrechia, M. Veillard, F. Puisieux, D. Labarre, Biomaterials 17, 1575 (1996)

28. I. Casinos, Polymer 33, 1304 (1992)

29. L. Bromberg, S. Deshmukh, M. Temchenko, L. lourtchenko, V. Alakhov, C. Alvarez-Lorenzo, R. Barreiro-Iglesias, A. Concheiro, A. Hatton, Bioconjugate Chem. 16, 626 (2005)

30. R. Motokawa, S. Koizum, T. Hashimoto, M. Annaka, T. Nakahira, Macromolecules 43, 752 (2010) 